Original Research Article

\title{
Automatic frontal sinus recognition in computed tomography images for person identification
}

\author{
Luis A. de Souza Jr. ${ }^{a},{ }^{*}$, Aparecido N. Marana ${ }^{a}$, Silke A.T. Weber ${ }^{b}$ \\ a São Paulo State University (UNESP), Department of Computing, Faculty of Sciences, Bauru, SP, Brazil \\ ${ }^{\mathrm{b}}$ São Paulo State University (UNESP), Department of Ophthalmology and Otorhinolaryngology, Botucatu Medical School, Botucatu, SP, Brazil
}

\section{A R T I C L E I N F O}

\section{Article history:}

Received 12 April 2017

Received in revised form 9 September 2017

Accepted 15 March 2018

Available online 23 March 2018

\section{Keywords:}

Person identification

Biometrics

Frontal sinus recognition

Image segmentation

Computed tomography

\begin{abstract}
A B S T R A C T
In many cases of person identification the use of biometric features obtained from the hard tissues of the human body, such as teeth and bones, may be the only option. This paper presents a new method of person identification based on frontal sinus features, extracted from computed tomography (CT) images of the skull. In this method, the frontal sinus is automatically segmented in the CT image using an algorithm developed in this work. Next, shape features are extracted from both hemispheres of the segmented frontal sinus by using BAS (Beam Angle Statistics) method. Finally, $L_{2}$ distance is used in order to recognize the frontal sinus and identify the person. The novel frontal sinus recognition method obtained $77.25 \%$ of identification accuracy when applied on a dataset composed of 310 CT images obtained from 31 people, and the automatic frontal sinus segmentation in CT images obtained a mean Cohen Kappa coefficient equal to 0.8852 when compared to the ground truth (manual segmentation).
\end{abstract}

(c) 2018 Elsevier B.V. All rights reserved.

\section{Introduction}

Biometric identification refers to the use of physical or behavioral characteristic to determine the person identity. In order to be used for person biometric identification a physical or behavioral characteristic must have some properties such as: universality, uniqueness, permanence, and acceptability [1].

Fingerprints and faces characteristics are frequently used for person biometric identification in commercial, banking, and government applications [1]. However, for the identification of deceased people, in forensic applications, these characteristics are not always available, because they derive from soft tissues of the human body that decomposes soon after death. In these cases, DNA features could be an alternative. However, in carbonized bodies, for instance, the characteristics of DNA cannot always be recovered [2]. Besides, DNA analysis is very expensive for applying to every case and it is not reliable in some cases as dry bones [3]. Therefore, in many human identification situations, the use of biometric features extracted from the bones, the hardest tissues of the human body, such as the frontal sinus features, may be the only option.

Frontal sinus consists of a paired irregularly shaped loculated cavity, located in the frontal bone [4]. The uniqueness property of the frontal sinus was first observed by Zuckerkandl, in 1875, when

\footnotetext{
* Corresponding author.

E-mail address: luis.souza@fc.unesp.br (L.A. d. Souza).
}

he called attention to its asymmetric morphology [5]. In 1927, Culbert and Law described the first human identification through morphological analysis of frontal sinus that was accepted in a United States court of law [6]. In 1943, Schuller was the first to suggest the possibility of identifying a deceased person by comparing frontal sinus radiography images [7]. In the beginning of 21st century, Ribeiro [8] and Kirk, Wood and Goldstein [9] reported positive results in skeletal identification using frontal sinus information extracted from radiography (X-ray) images.

The frontal sinus asymmetry, among other particular characteristics, has motivated several studies in the last few decades aiming people identification through the analysis of frontal sinus measurements obtained from X-ray films. Many of these studies have established that the highly variable radiographic pattern of the frontal sinus is unique to every individual, even among monozygotic twins [7-10].

The frontal sinus is increasingly being used for postmortem human identification. In 2007, Tatlisumak et al. [3] defined a system for the identification of unknown bodies by using computed tomography images of frontal sinus. This system, called FSS, included simple features as $\mathrm{F}$ (presence or absence of frontal sinus), S (intersinus and intrasinus septum) and S (scalloping). Measurements selected for this study were width, height, anteroposterior length, total width of two sinuses, the distance between the highest points of the two sinuses and the distance of each sinus to its maximum lateral limit. All the features and measurements were coded according to the FSS system defined for 
each case and coded formulas were compared. At least $93 \%$ of the formulas could be eliminated for a case by using FSS system.

In 2015, Soares et al. [11] performed a study to evaluate the applicability of human identification parameters, established by Tatlisumak et al. [3], using cone-beam computed tomography tridimensional reconstruction and extraoral radiographs of the frontal sinus. They concluded that, despite some advantages of using X-ray, such as the low costs, comparison of radiographic images has limitations because it is a subjective test that depends on the expert's experience and the radiographic image can vary significantly according to the incidence of the X-ray beam. They recommend the use of $\mathrm{CT}$ imaging examination for proper study of the paranasal sinuses, since it enables the acquisition of sectional images, three-dimensional scans of the sinuses, and eliminates the structural overlapping observed in X-ray images.

In 2015, Hashim et al. [12] made a study that tested the practical relevance of prescribing superimposition by superimposing anteand-post-mortem frontal sinus patterns recorded in real case situations and ante-and-post-mortem of frontal sinus patterns in simulated cases, recorded using archived skulls, resulting in a perfect match of radiographs from the same skull only when the orientation of the skull obtained for the first (simulated antemortem) recording is unaltered for the second (simulated postmortem) recording. According to the authors, for the real life cases, normally the superimposition is not possible.

In 2016, Rabelo et al. [13] studied the applicability of the FSS system proposed by [3] in order to identify persons, by means of the frontal sinus measures acquired from frontal and lateral cephalometric radiographs. They reported good results.

Researches also have been carried out in order to identify gender by analyzing measurements of the frontal sinuses obtained from X-ray films. Belaldavar et al. [14], for instance, concluded that the area, height and width of the frontal sinuses are, in average, bigger in men than in women. Uthman et al. [15] and Carvalho et al. [16], conducted experiments focusing gender discrimination and human identification using CT images and structures of the paranasal sinuses (frontal sinuses and other cranial structures).

In general, these previous works are based on manual or semiautomatic frontal sinus segmentation methods. The review by Xavier, Terada and Silva [17] showed that there were no fully automatic frontal sinus segmentation method proposed in literature so far.

Another characteristic of previous works is that they are based on X-ray images. However, computed tomography (CT) images allow better frontal sinuses segmentation and recognition than $\mathrm{X}$ ray images because of the better contrast provided by the $\mathrm{CT}$ technology. Fig. 1 shows an X-ray image and a computed tomography (CT) image of two distinct individuals, with the

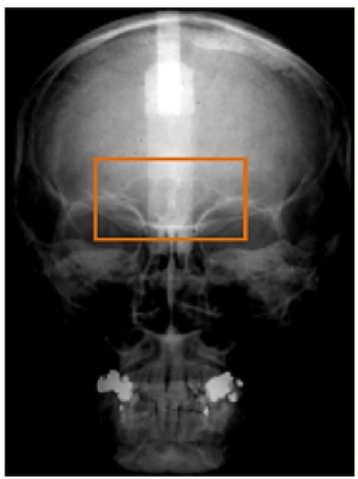

(a)

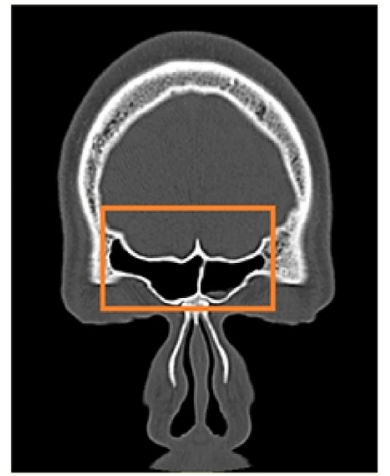

(b)
Fig. 1. Frontal sinuses enclosed by rectangles: (a) X-ray image and (b) computed tomography image. frontal sinuses enclosed by rectangles. One can observe that the CT image (right) allows an easier and better analysis of the frontal sinus structures, when compared to the X-ray image (left). Besides, CT scans produce sequences of images, allowing 3D analysis of the frontal sinus structures.

The goal of our work is to propose and develop a fully automatic and robust method for frontal sinus segmentation and recognition using computed tomography images to be used for person identification.

\section{Frontal sinus}

Frontal sinus consists of a paired irregularly shaped loculated cavity, located in the frontal bone, which communicate with the nasal fossa via the infundibulum [4]. This cavity develops embryonically from an ethmoidal cell and is not visible at birth. It starts developing during the second year of life, reaching its maximum size at the age of 20 .

As the frontal sinus remains stable even after death $[4,10]$ it is very suitable for post mortem person identification. The configuration of the frontal sinus is claimed to be unique for each individual $[4,7,9,10]$ and is controlled by genetic and environmental factors. Frontal sinus variations have been reported in the literature even for monozygotic twins $[4,10]$.

Fig. 2 shows three CT images of three different individuals. As one can observe in the red rectangles, the three frontal sinuses have very distinct and particular characteristics that can be used to identify the individuals.

\section{Proposed method}

The new method proposed in this work for person identification based on frontal sinus features extracted from computed tomography images has three main stages:

- Frontal sinus segmentation;

- Frontal sinus feature extraction; and

- Frontal sinus recognition.

\subsection{Frontal sinus segmentation}

The frontal sinus segmentation stage consists in automatically detecting in $\mathrm{CT}$ images the pixels belonging to the internal region of the frontal sinus.

The first step of this process is the binarization of the gray level CT image, which is carried out by replacing the original gray scale pixel values by 0 or 1 , according to a threshold value. This technique was chosen because it is fast and results in very good quality segmentation since $\mathrm{CT}$ images present high contrast in the frontal sinus region, as one can observe in Fig. 2 (black regions completely surrounded by white borders).

In our method, the threshold value used for binarization is automatically defined. Given $I$, an $m \times n$ CT image, its gray level histogram $H$ is calculated. Then, by considering the rightmost mode $\left(m_{r}\right)$ of $H$, cranial bones regions in the CT images can be easily detected since they are associated with the brighter regions of $I$. So, the threshold value $(t)$ of the binarization stage is taken as $80 \%$ of $m_{r}$.

Fig. 3(b) shows the logarithm of the gray level histogram $H$ of the CT image of Fig. 3(a), with the threshold $t$ represented by the vertical red line located at the left side of the rightmost mode of $H$. Fig. 3 (c) shows the binary image $B_{1}$ obtained automatically with the binarization process applied to the gray level CT images $I$ presented in Fig. 3(a).

After this first binarization, the next step is to find the region of interest (ROI) of the CT image, that is, the smaller rectangle that contains the frontal sinus. An initial ROI, called $R_{1}$, is taken as the 


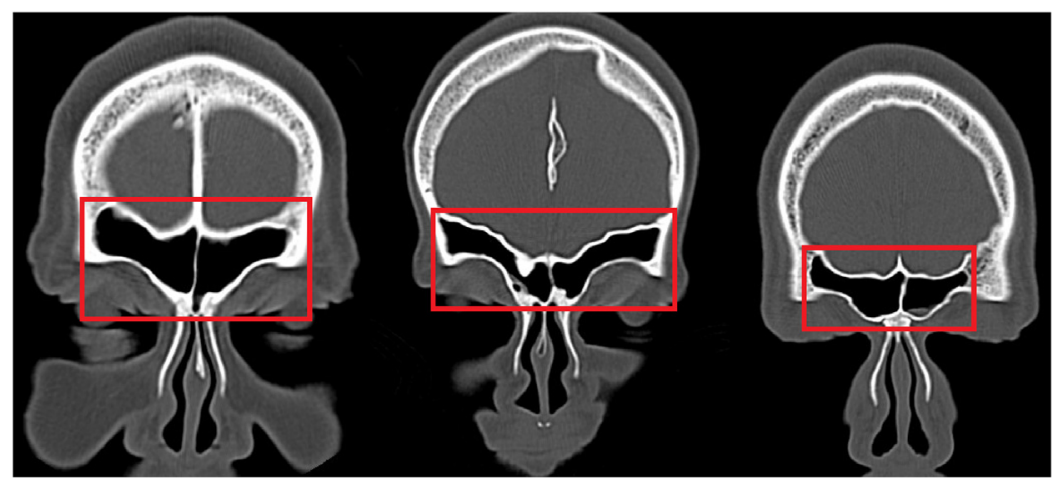

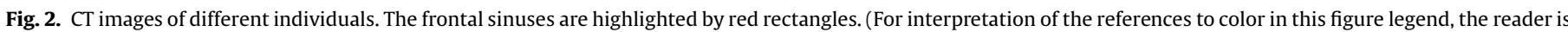
referred to the web version of this article.)

rectangle defined by the top and bottom straight lines found in the binary image $B_{1}$ by using the function $g$, defined in Eq. (1), and by the left and right straight lines found in the binary image $B_{1}$ by using the function $f$, defined in Eq. (2),

$g(i)=\sum_{j=1}^{n} B_{1}(i, j), \quad i=1, \ldots, m$.

$f(j)=\sum_{i=1}^{m} B_{1}(i, j), \quad j=1, \ldots, n$.

with $m$ and $n$ being the height and the width of $B_{1}$, respectively.

Fig. 4 shows the four straight lines that define the top, bottom, left, and right limits of $R_{1}$, found in the binary image $B_{1}$, by using the Eqs. (1) and (2).

The left and right straight lines correspond, respectively, to the leftmost and the rightmost columns of $B_{1}$ for what the function $f$ is different of zero, while the top and the bottom straight lines correspond, respectively, to the topmost and the bottommost rows of $B_{1}$ for what the function $g$ is different of zero.

One can observe in Fig. 4 that the ROI $R_{1}$ encloses the cranial region of the CT image. However, our goal is to find the smaller rectangle that encloses the frontal sinus. Then, a new ROI, called $R_{2}$, is calculated from $R_{1}$. A new image $I_{2}$ is calculated by cropping the image $I$, using the $R_{1}$ limits. From $I_{2}$, a new binary image, called $B_{2}$, is calculated considering as threshold the leftmost mode of the gray level histogram $H$ plus 1 . The binarization applied is described by Eq. (3).

$B_{2}(i, j)= \begin{cases}0, & \text { if } I_{2}(i, j) \geq \text { threshold } \\ 1, & \text { otherwise }\end{cases}$

In this second binarization process, all pixels with gray levels values equal or above the threshold are set to 0 (black), while all pixels with gray level values below to the threshold are set to 1 (white).

Fig. 5(a) shows the image $I_{2}$ obtained by cropping the image $I$, presented in Fig. 3(a), using the ROI $R_{1}$ limits. Fig. 5(b) shows the new binary image $B_{2}$ obtained after the second binarization process.

In order to find the bottom and top limits of the white structure inside $B_{2}$, the function $g$ (Eq. (1)) is applied to $B_{2}$.

It is possible to observe in Fig. 6 that at the beginning, as $\mathrm{i}$ increases from 0 to $m$, the values of $g(i)$ decreases, due the round shape of the skull that forms a white region in the left and right top corners of the binary image $B_{2}$, or keep constant until $i$ reaches the value of the first row (top row) that intersects the frontal sinus region. After that, the values of $g(i)$ start increasing, as the value of $i$ increases. So, we are able to identify the top limit of the $R_{2}$ region by identifying the leftmost value of $i$, that we called $x_{1}$, for what $g(i)$ starts growing. On the other side, as $i$ decreases from $m$ to 0 , the values of $g(i)$ decreases, due the round shape of the skull that forms a white region in the left and right bottom corners of the binary image $B_{2}$, or keep constant until $i$ reaches the value of the first row (bottom row) that intersects the frontal sinus (or other nearby cavity) region. After that, the values of $g(i)$ start increasing, as the value of $i$ decreases. So, we are able to identify the bottom limit of the $R_{2}$ region by identifying the rightmost value of $i$, that we called $x_{2}$, for what $g(i)$ starts growing.

Fig. 7 shows the binary image, $B_{3}$, obtained by cropping $B_{2}$, using the $R_{2}$ limits. One can observe in Fig. 7 that $B_{3}$ is composed by a number of white regions corresponding to the frontal sinus and other structures. Because some of these regions do not belong to the frontal sinus, a new region of interest, called $R_{3}$, is found containing only the white regions of the frontal sinus. The algorithm used for this purpose is presented in Algorithm 1.

Algorithm 1. Algorithm to calculate the ROI $R_{3}$.

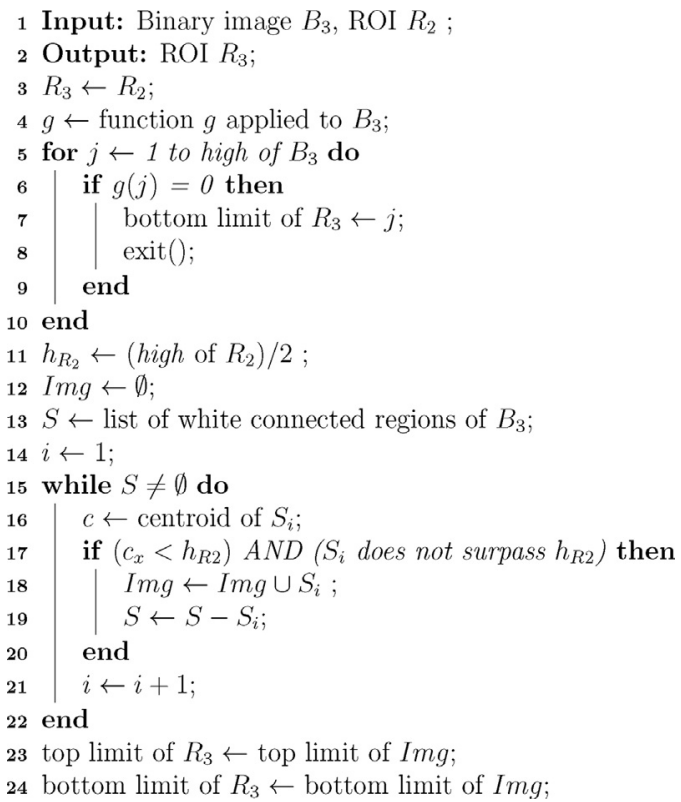

As one can observe in Algorithm 1 there are two ways to define the ROI $R_{3}$. Initially $R_{3}$ is taken as $R_{2}$ (line 3 ). Then, the function $g$ (Eq. (1)) is applied to the binary image $B_{3}$ (line 4 ). Next, if there is a row $j$ in $R_{3}$ such as $g(j)$ is equal do zero, then, the bottom limit of $R_{3}$ is found and the algorithm ends (lines 5-10). Otherwise, the 


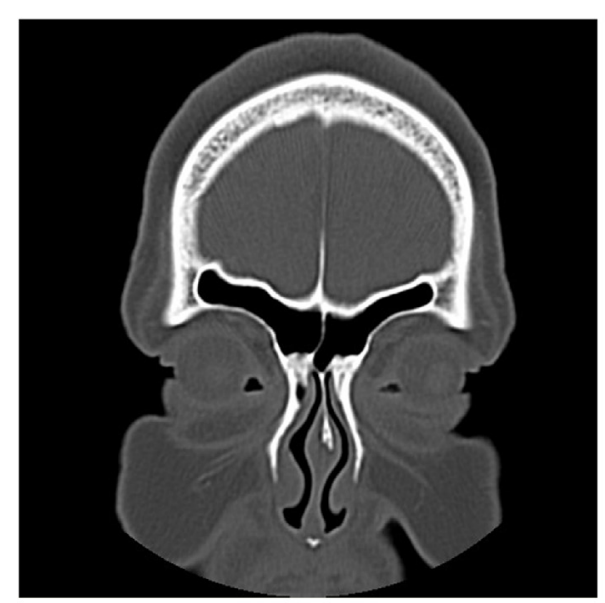

(a)

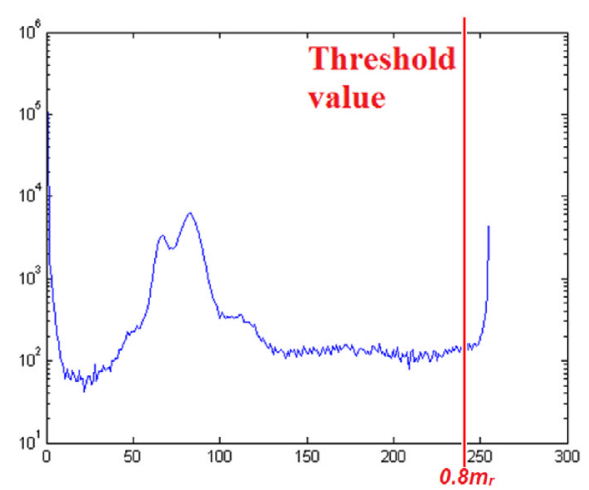

(b)

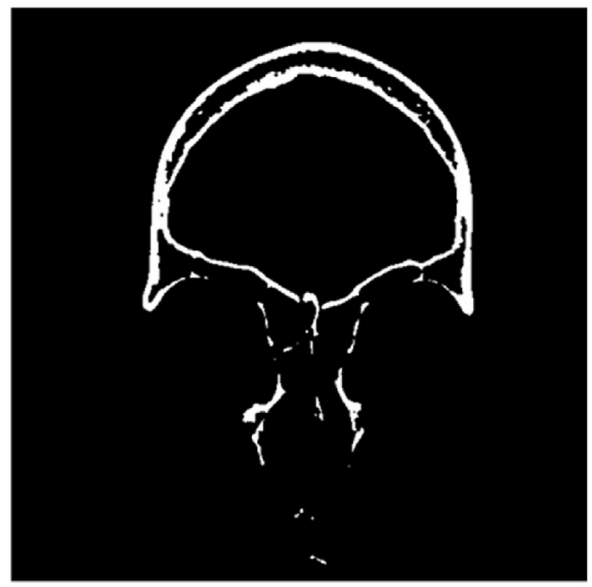

(c)

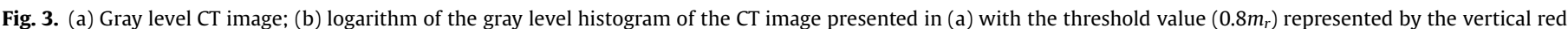

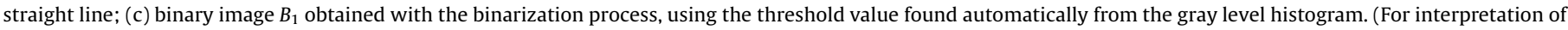
the references to color in this figure legend, the reader is referred to the web version of this article.)

central row of $B_{3}$, called $h_{R_{2}}$, is calculated (line 11) and the white connected regions of $B_{3}$ are stored into a list $S$ (line 13). Next, while a white region $S_{i}$ exists in $S$, its centroid is calculated (line 16). If such centroid is above $h_{R_{2}}$ and the $S_{i}$ structure is completely above $h_{R_{2}}$, this region is added to the new image Img being created with the white connected regions that compose the frontal sinus (lines 15-22). Finally, the final top and bottom limits of $R_{3}$ are taken as the top and the bottom limits of Img. The left and the right limits of $R_{3}$ are taken as the left and the right limits of $R_{2}$.

Fig. 8 shows an example of ROI $R_{2}$ defined in the first part of Algorithm 1 (line 3-10). Fig. 9 shows an example of ROI $R_{2}$ that contains regions that do not belong to the frontal sinus. In this case, the second part of Algorithm 1 (lines 11-24) is executed. In the example presented in Fig. 9, one can observe that the horizontal straight line divides $R_{2}$ in two parts. In this figure, the regions are colored with different colors. The blue regions belong to the frontal sinus. The other regions (yellow, red, orange and green) represent other cranial structures. Because the centroids of red region are below $h_{R_{2}}$, they are removed. Green regions are too small, then they are removed as well. Yellow regions are located in the border of the ROI, so they are removed too. Although the centroid of orange region is above $h_{R_{2}}$, it is not completely above $h_{R_{2}}$, then this region is discarded. All these steps are necessary because it is difficult to find the bottom limit of the ROI $R_{3}$ due to the great variances of the bone structures in this region of the cranium.
Fig. 10 illustrates the automatic frontal sinus ROI detection method in CT images proposed in our work. Initially, the ROIs $R_{1}$ (yellow rectangle) and $R_{2}$ (green rectangle) are calculated in order to guide the final ROI detection, $R_{3}$ (red rectangle).

After finding the ROI $R_{3}$, the goal is to segment both left and right frontal sinus hemispheres, which is carried out by using the centroids of each connected components inside $R_{3}$. These centroids are used to classify each component as belonging to the left or to the right side of ROI $R_{3}$. Fig. 11(a) and (b) show the binary images containing the left and right hemispheres of the frontal sinus, respectively, obtained from the ROI $R_{3}$.

Fig. 12 presents a diagram that summarizes all steps of the proposed method for automatic segmentation of the left and right hemispheres of the frontal sinuses from CT images.

\subsection{Frontal sinus feature extraction}

The frontal sinus descriptor adopted in our work is the Beam Angle Statistics (BAS) shape descriptor, proposed by Arica and Vural [18], extracted from the left and right frontal sinus hemispheres. BAS descriptor was chosen because it presents very good results in a number of shape analysis and recognition problems [19,20].

The Beam Angle Statistics (BAS) method is a consistent twodimensional (2D) shape descriptor based on the 2D shape 


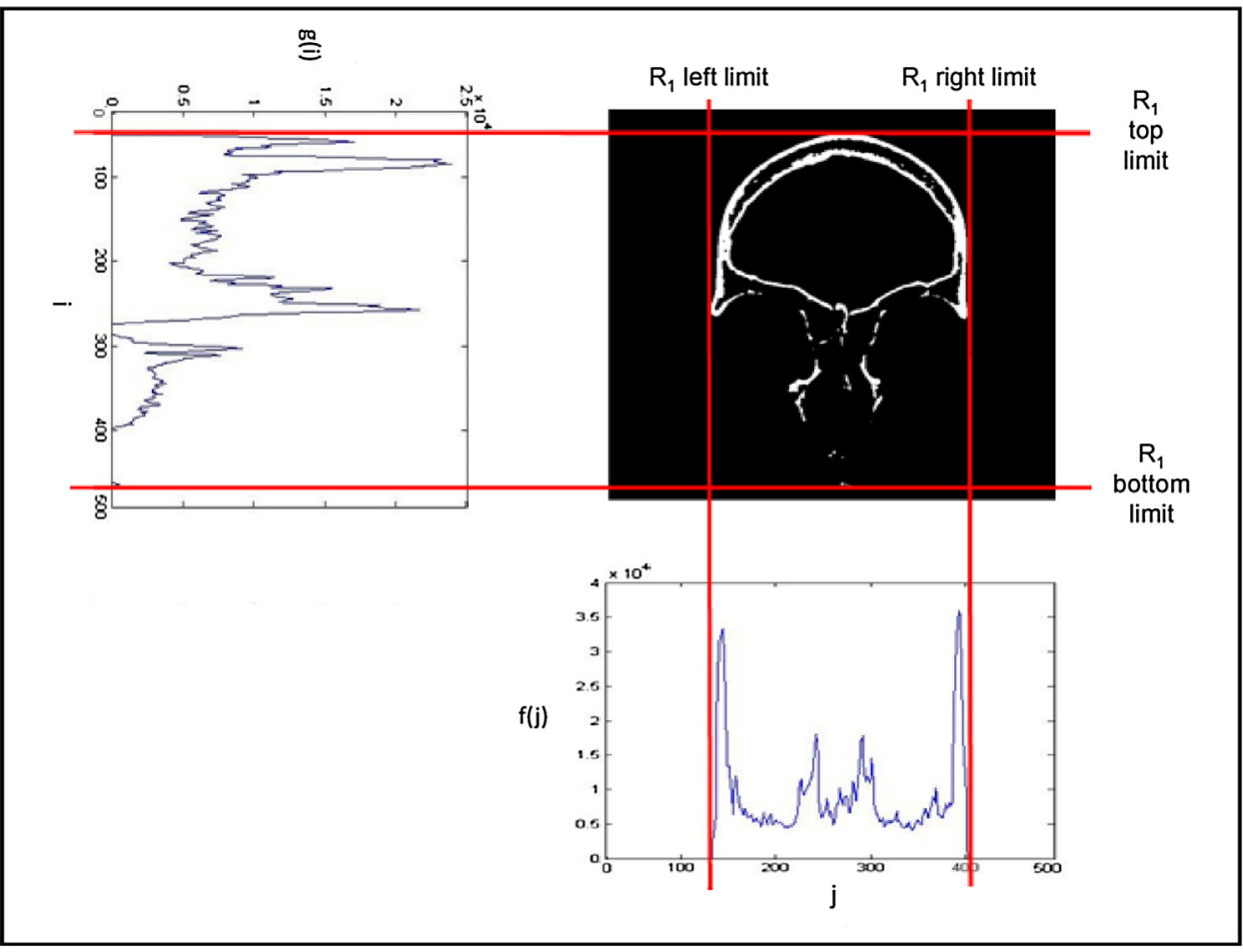

Fig. 4. Illustration of the method proposed to find the ROI $R_{1}$.

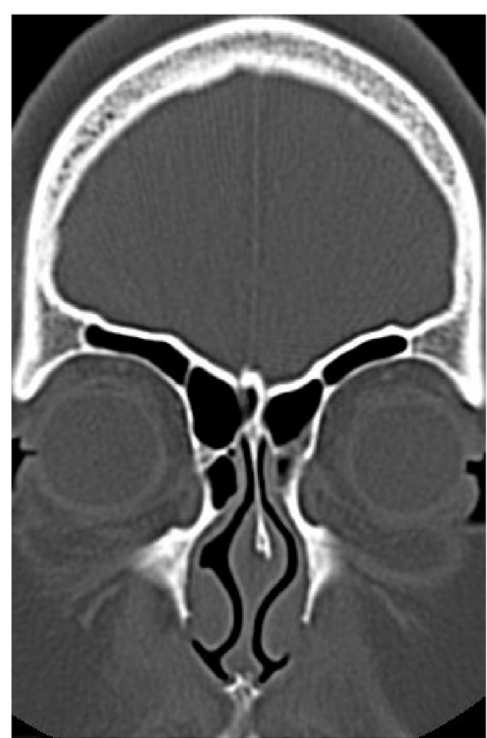

(a)

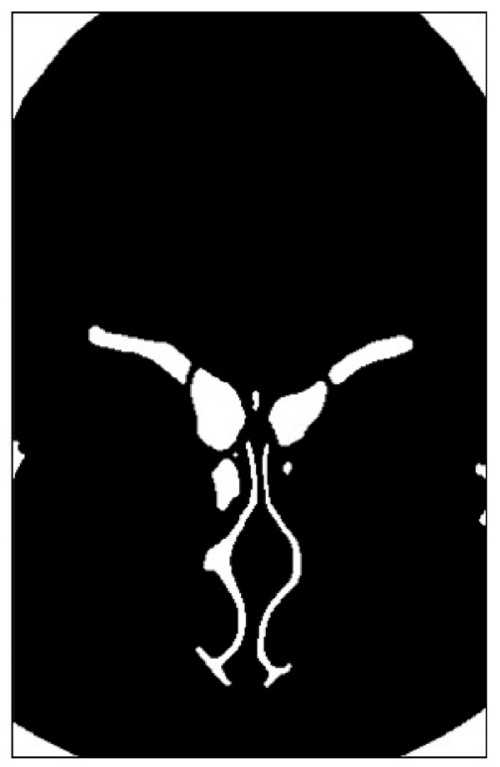

(b)

Fig. 5. (a) Image $I_{2}$ obtained by cropping the image $I$ using $R_{1}$ limits; (b) binary image $B_{2}$, the binarization of $I_{2}$.

representation. In this method an object's boundary $O B$ is represented as a connected sequence of points $p_{i}=\left(x_{i}, y_{i}\right), i=1$, $2, \ldots, n$, where $n$ is the number of points that belongs to the object's boundary, and $p_{i}=p_{i+n}$, i.e., the sequence of points is circular.

Adopting a boundary point $p_{i}$ and considering the angle $c_{j}(i)$ between the two vector originating at $p_{i-j}$ and $p_{i+j}$, with $j$ varying from 1 to $n / 2$, as a random variable $\left(C_{i}\right)$, it can be associated with $p_{i}$ the first, second and third moments of this variable (mean, variance and skewness, respectively). This process is repeated for all points belonging to the $O B$ boundary, resulting in the first, second and third moment-based functions (1D functions). Then, the feature vector is built by sampling the 1D function at $k$ equally spaced positions of the boundary.

Since the segmented frontal sinus hemispheres in the first stage may be not connected, as one can observe in Fig. 11, a morphological closing operation is applied. After this closing operation, BAS descriptors are extracted from the fully connected left and right frontal sinus hemispheres. The feature vector of a CT scan is finally defined as the concatenation of all BAS descriptors 


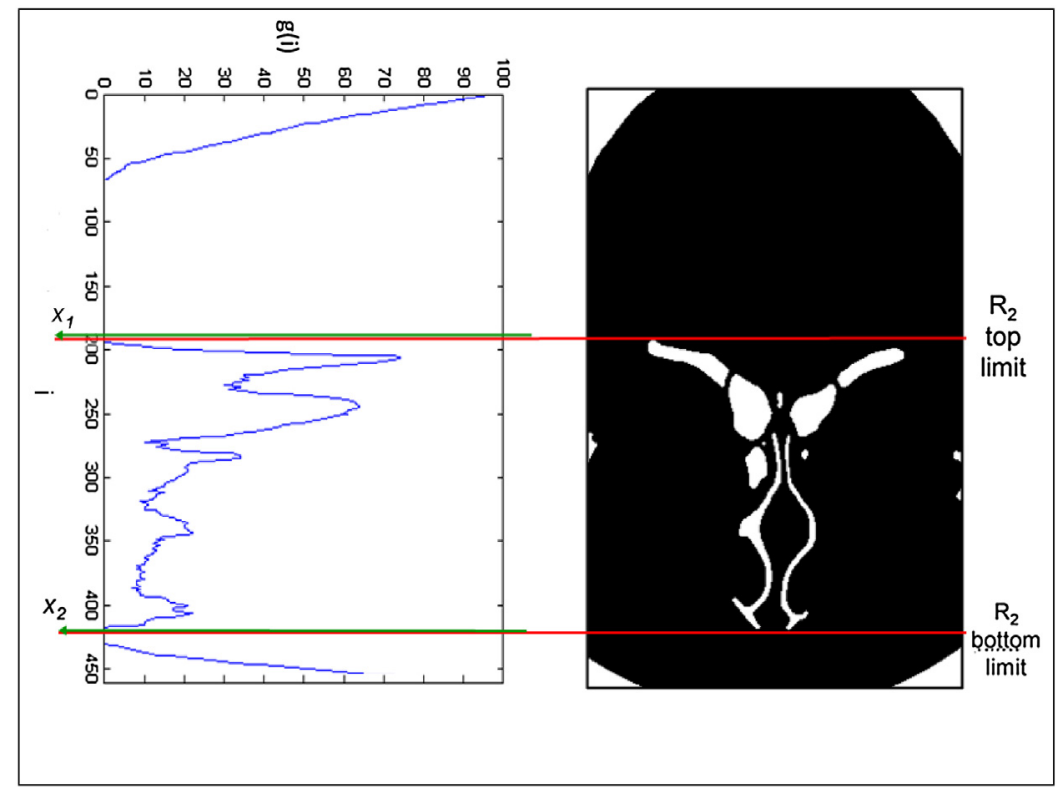

Fig. 6. Illustration of the proposed method to find the ROI $R_{2}$.

extracted from the left and the right hemispheres of all images that compose the CT scan.

\subsection{Frontal sinus recognition}

In the recognition stage of our method, the frontal sinus feature vectors of the probe $(\Pi)$ and gallery $(\Gamma)$ CT images are compared,

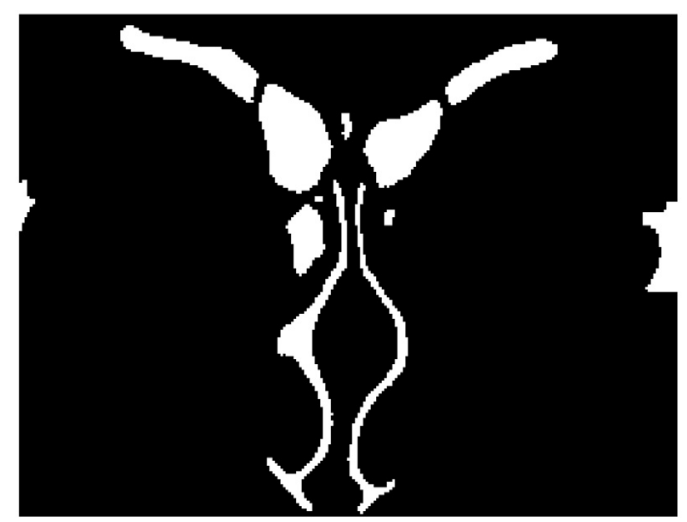

Fig. 7. Binary image $B_{3}$.

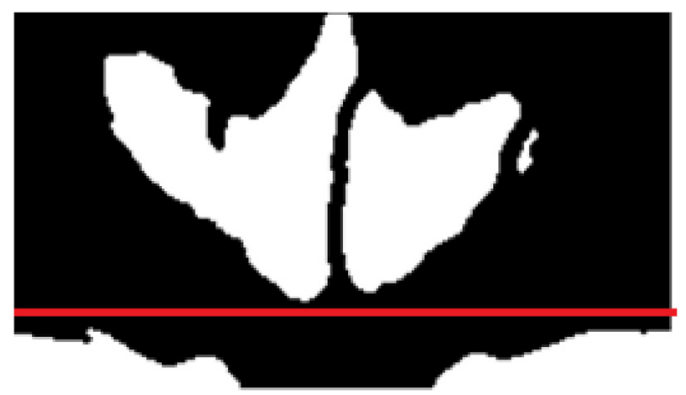

Fig. 8. Example of ROI $R_{2}$ for what the application of the first part of Algorithm 1 (lines 3-10) is enough to find the bottom limit (red line) of the ROI $R_{3}$ containing only the frontal sinus regions. (For interpretation of the references to color in this figure legend, the reader is referred to the web version of this article.) using the $L_{2}$ distance (Euclidian distance), according to Eq. (4).

$$
D(\Pi, \Gamma)=\sqrt{\sum_{i=1}^{k}(\Pi(i)-\Gamma(i))^{2}}
$$

where $k$ is the feature vector size.

Given a query frontal sinus CT sequence of images, its classification is performed by comparing the similarity of its feature vector, $\Pi$, with each gallery feature vector, $\Gamma$, associated to each individual in the database. The assigned identity will be the one associated with the gallery identity whose feature vector $\Gamma$ has minimum $L_{2}$ distance to the query feature vector П. Fig. 13 illustrates the recognition stage of our method.

\section{Assessment protocol}

This section presents the ground truth and the agreement measure between the ground truth and the automatic segmentation obtained by our algorithm. It also presents the approach adopted in our experiments for obtaining the genuine and impostor matching score distributions, as well as the ROC

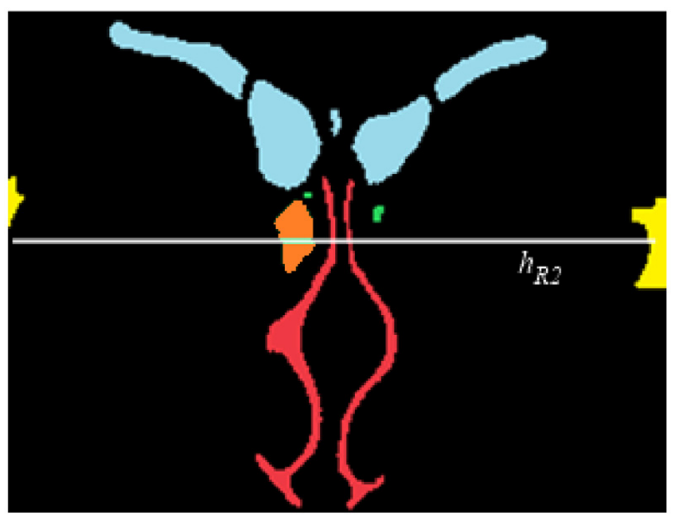

Fig. 9. Example of ROI $R_{2}$ for what the application of the second part of Algorithm 1 (lines 11-24) is necessary to find the correct bottom limit of the ROI $R_{3}$, keeping only the frontal sinus regions. (For interpretation of the references to color in this figure legend, the reader is referred to the web version of this article.) 


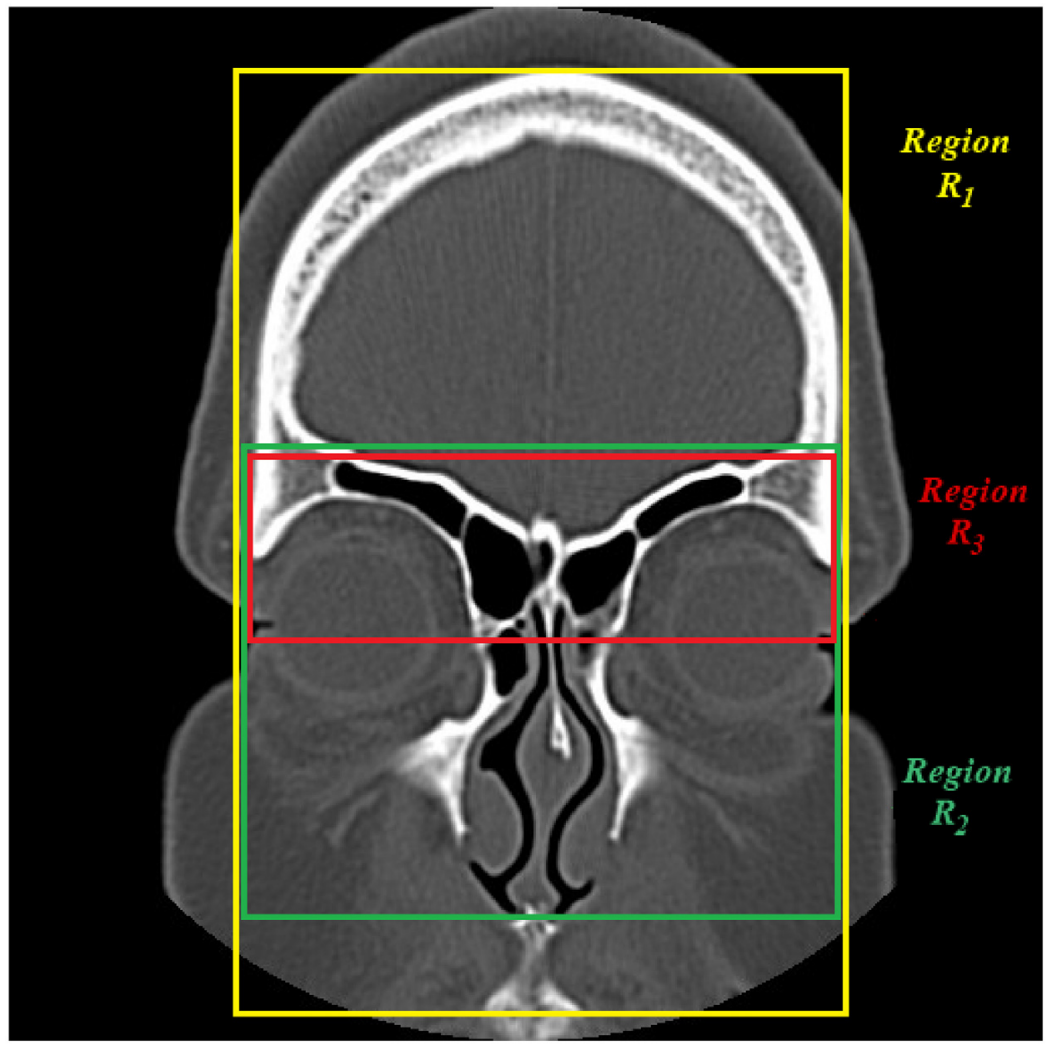

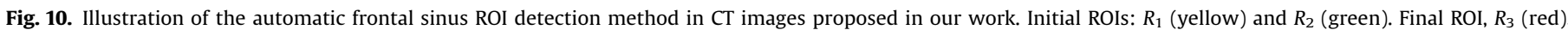
containing the frontal sinus.

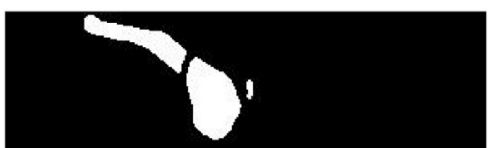

(a)

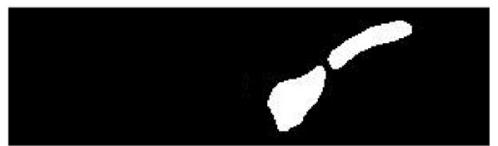

(b)

Fig. 11. (a) Right hemisphere of the frontal sinus detected inside the ROI $R_{3}$; (b) left hemisphere of the frontal sinus detected inside the ROI $R_{3}$.

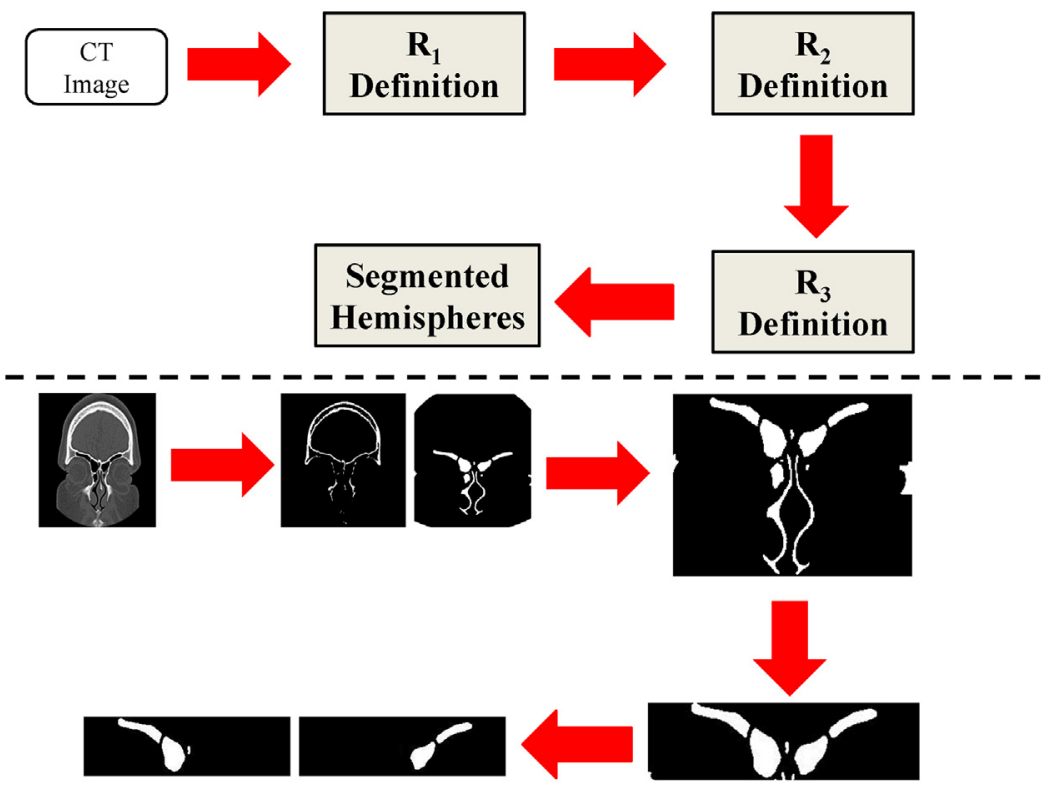

Fig. 12. Diagram of the proposed method for automatic CT frontal sinus image segmentation. 


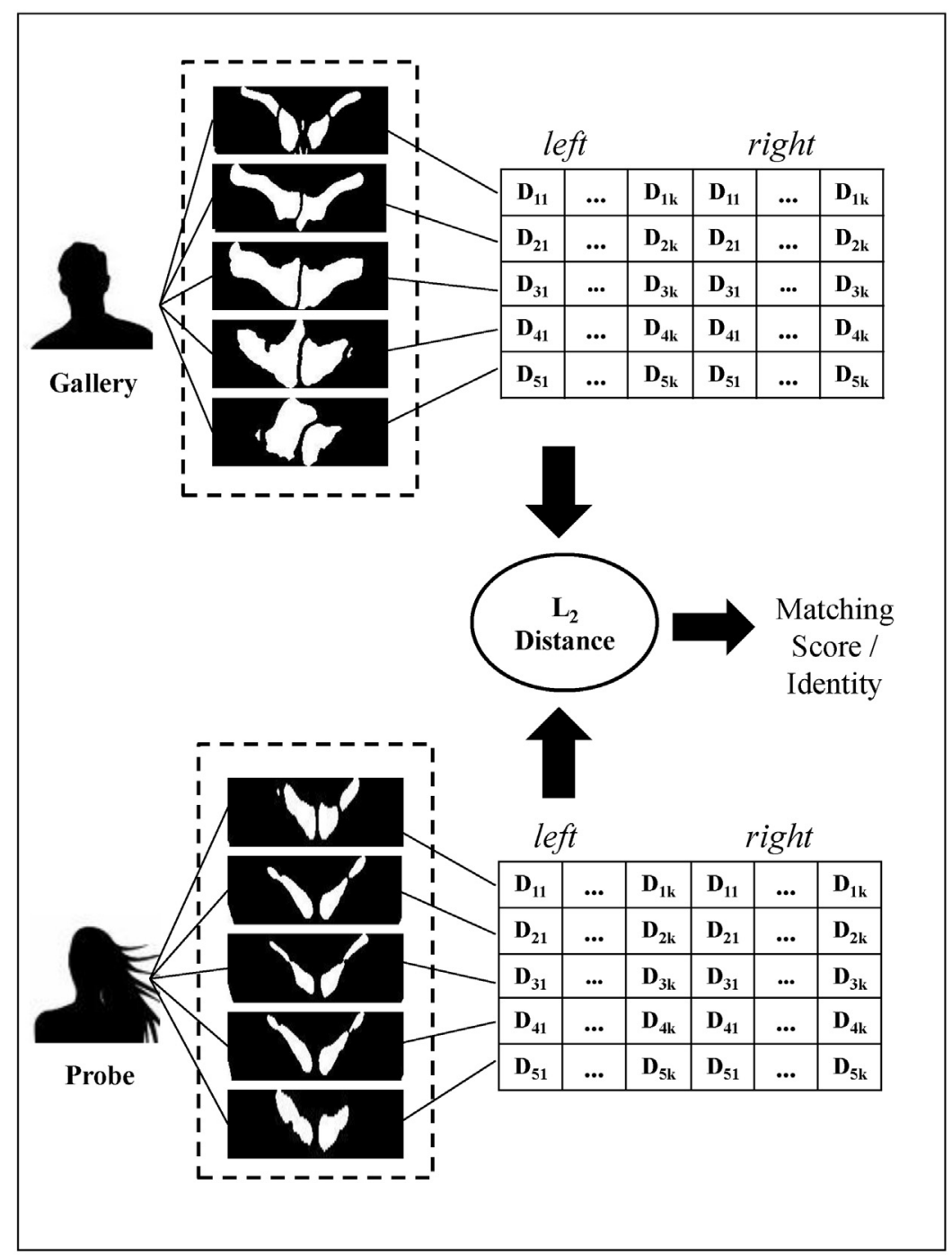

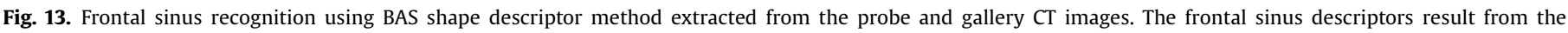

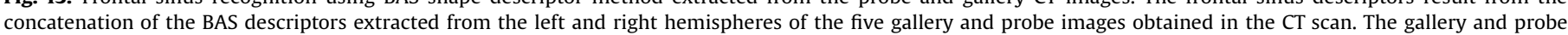
descriptors are compared by using the $L_{2}$ distance.

(Receiver Operating Characteristic) and CMC (Cumulative Match Characteristic) curves, used to assess the performance of our frontal sinus automatic recognition method.

\subsection{Ground truth}

In order to evaluate the performance of the automatic frontal sinus segmentation method proposed in this work, it was necessary to use a ground truth dataset. Therefore, we carried out the manual segmentation of all frontal sinus images of our database. To perform such manual segmentation, the Image ${ }^{1}$ software was used. With this software, upon a new layer over the image, the frontal sinus border was manually lined out by using the mouse device. The manual segmentation was evaluated and validated by a Professor, expert in Radiology and Head and Neck Surgery, of the Botucatu Medical School, UNESP, campus of Botucatu, São Paulo, Brazil.

\footnotetext{
1 https://imagej.nih.gov/ij/.
}

\subsection{Agreement measure of manual and automatic frontal sinus} segmentation

In order to evaluate the results obtained by our automatic frontal sinus segmentation method, the Cohens Kappa coefficient was used as a measure of agreement with the manual segmentation (our ground truth dataset).

The Cohens Kappa coefficient is given by [21]:

$$
K A P=\frac{f_{a}-f_{c}}{N-f_{c}}
$$

where $N$ is the total number of observations, $f_{a}$ and $f_{c}$ are defined as:

$f a=T P+T N$

$f C=\frac{(T N+F N)(T N+F P)+(F P+T P)(F N+T P)}{N}$

The terms in the Eqs. (6) and (7) are expressed by the overlap coordinates: True Positive (TP), True Negative (TN), False Positive 

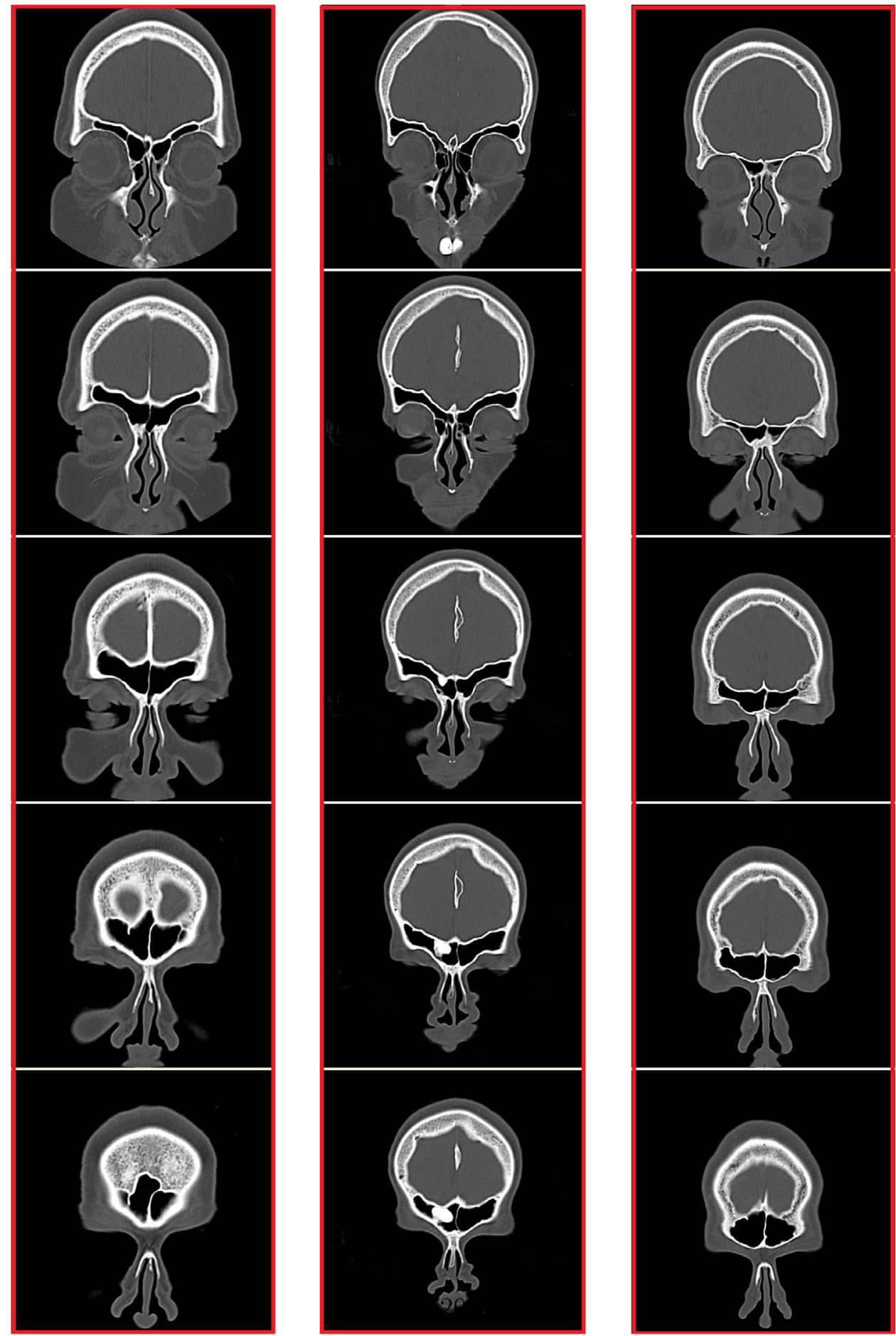

Fig. 14. Three sequences of CT scans of frontal sinus computed tomography (FSCT) database. Each column represents a different person.

(FP) and False Negative (FN), defined as:

$$
\begin{aligned}
& T P=\sum_{r=1}^{|x|} \min \left(f_{a}^{1}\left(x_{r}\right), f_{m}^{1}\left(x_{r}\right)\right) \\
& F P=\sum_{r=1}^{|x|} \max \left(f_{a}^{1}\left(x_{r}\right)-f_{m}^{1}\left(x_{r}\right), 0\right) \\
& T N=\sum_{r=1}^{|x|} \min \left(f_{a}^{2}\left(x_{r}\right), f_{m}^{2}\left(x_{r}\right)\right)
\end{aligned}
$$$$
\text { (8) } \quad F N=\sum_{r=1}^{|x|} \max \left(f_{a}^{2}\left(x_{r}\right)-f_{m}^{2}\left(x_{r}\right), 0\right)
$$

where $f_{a}^{1}$ is the foreground values of the automatic segmentation result (frontal sinus), $f_{a}^{2}$ is the background values of the automatic segmentation result, $f_{m}^{1}$ is the foreground values of the manual segmentation result (frontal sinus), and $f_{m}^{2}$ is the background values of the manual segmentation result. 


\section{Automatic versus Manual Segmentation}

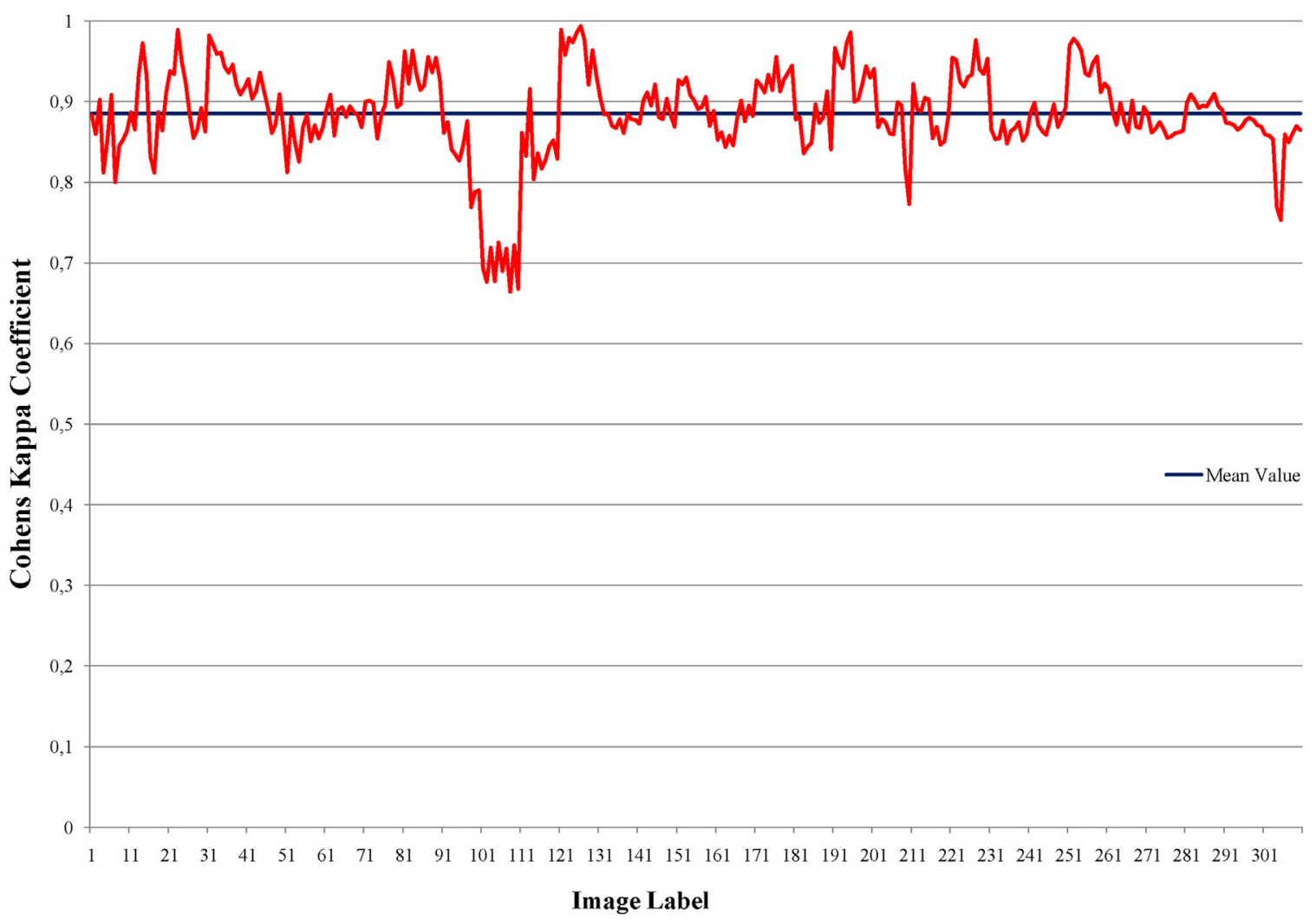

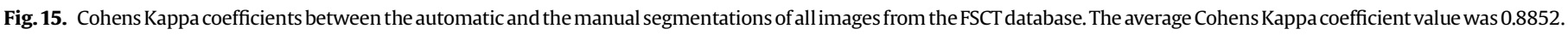

\subsection{Genuine and impostor matching score distributions}

The genuine and impostor matching score distributions were calculated by comparing the frontal sinus BAS descriptors, according to the following protocol:

- Genuine matching: Frontal sinus descriptors extracted from different $\mathrm{CT}$ images of the same individual are compared and the matching scores are calculated.
- Impostor matching: Frontal sinus descriptors extracted from CT images of different individuals are compared and the matching scores are calculated.

\subsection{Identification performance}

After obtaining the genuine and the impostor matching scores, the respective genuine and impostor matching score distribution

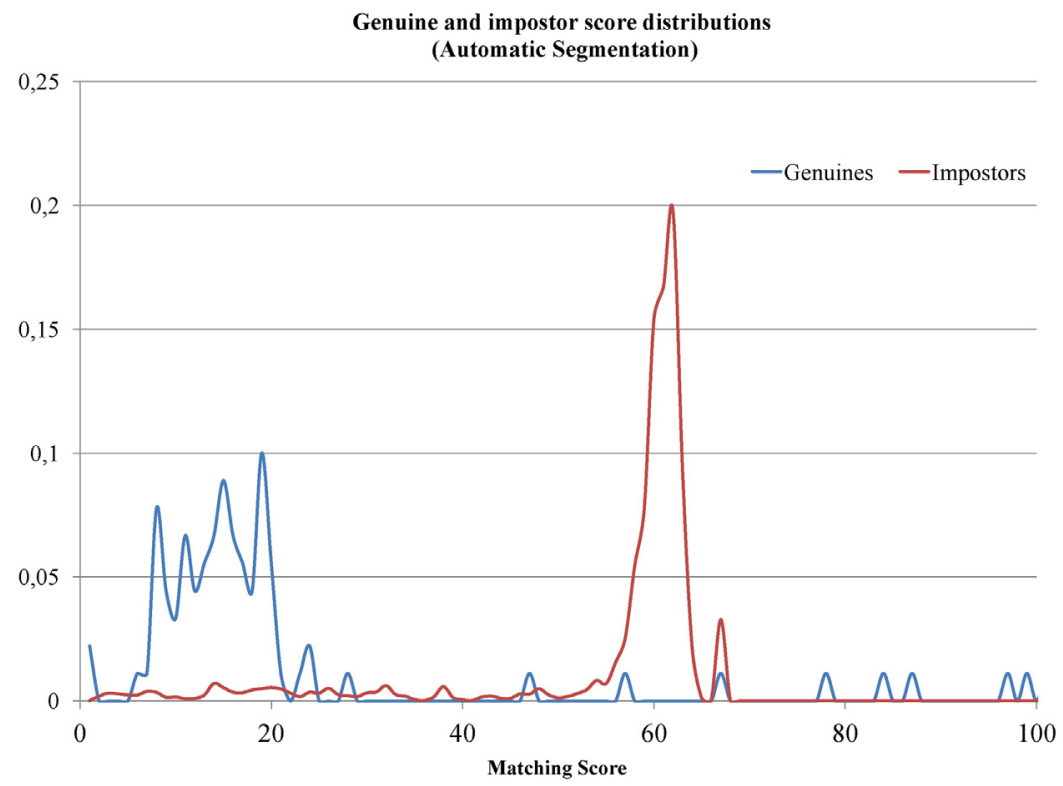

Fig. 16. Genuine and impostor score distributions obtained by the proposed method using BAS shape descriptors. 
ROCs

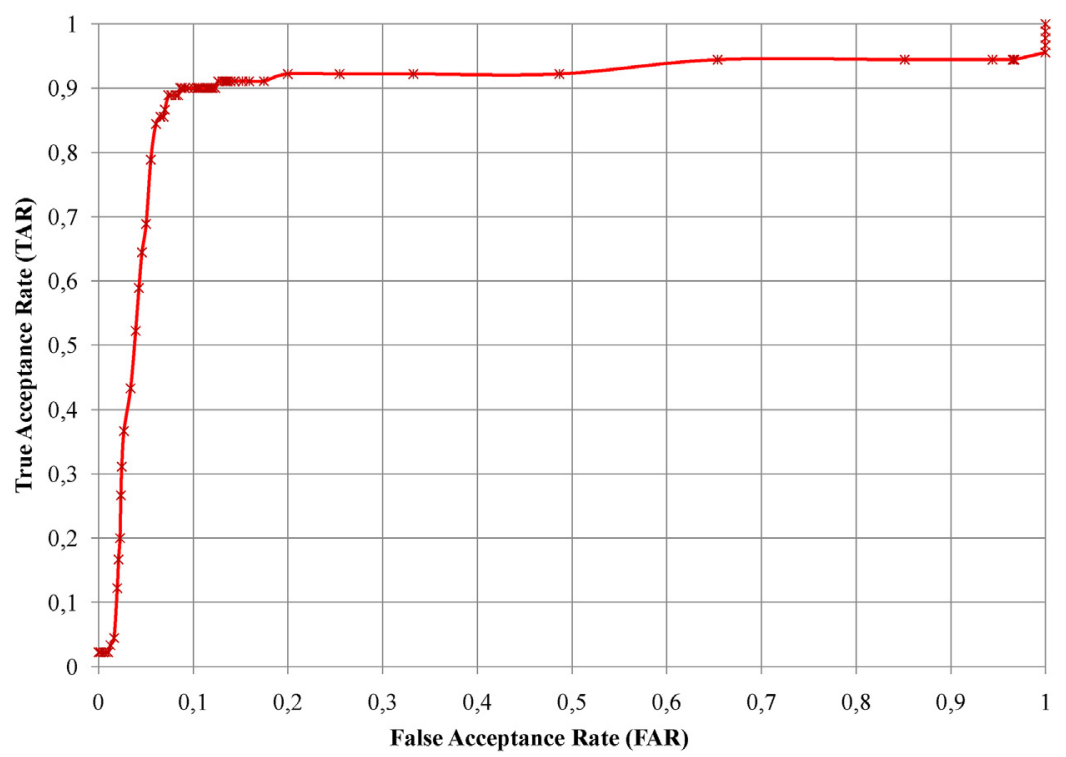

Fig. 17. ROC curve obtained by the proposed method using BAS shape descriptors.

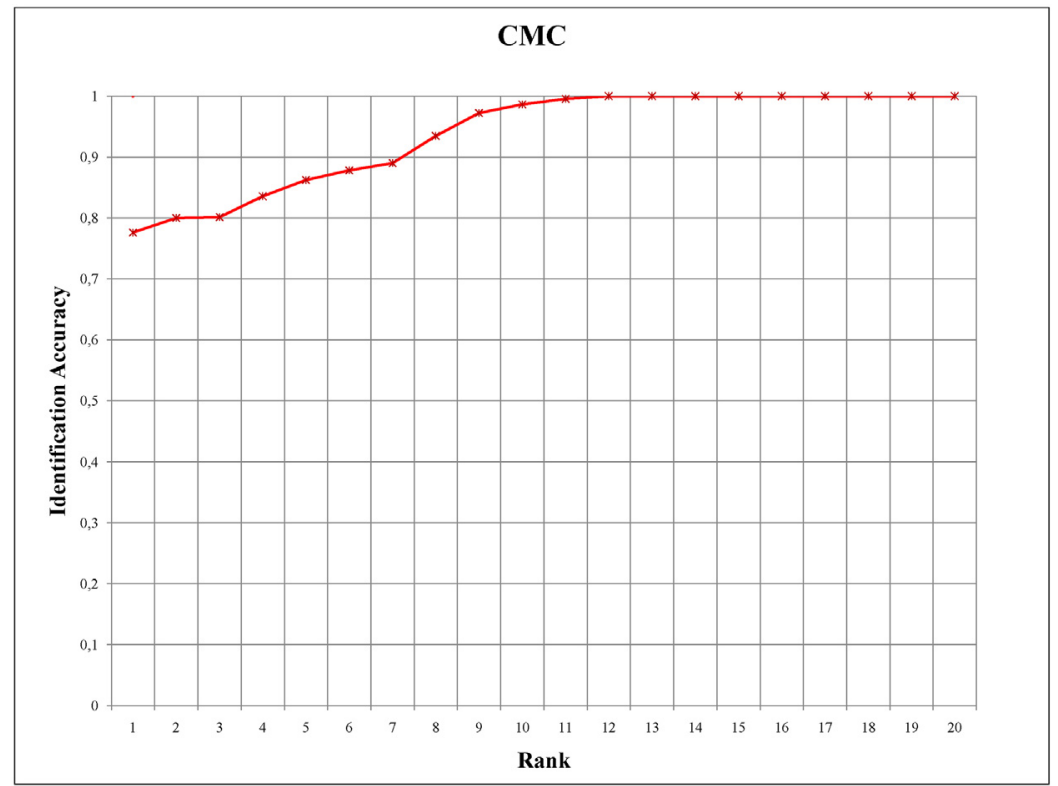

Fig. 18. CMC curve obtained by the proposed method using BAS shape descriptors.

curves are calculated, as well as the Equal Error Rate (EER), the ROC curve and the CMC curve.

The EER refers to the score value (threshold) for which the false rejection rate (FRR) is equal to the false acceptance rate (FAR).

The performance of a verification system is summarized using ROC curve, which is the plot of the false acceptance rate (FAR) against the true acceptance rate (TAR $=1-$ FRR).

The performance of a closed-set identification system is summarized using CMC curve, which presents the accuracy in the identification mode for different rank values. In order to calculate the CMC curve, each probe biometric sample is compared against all gallery samples. Then, the resulting matching scores are sorted and ranked. The CMC curve determines the rank at which a true match occurs.

\section{Experimental results}

This section presents the frontal sinus computed tomography (FSCT) database used in this work and the experimental results obtained with the new automatic frontal sinus recognition method. 


\subsection{Frontal sinus computed tomography database}

The frontal sinus computed tomography (FSCT) database used in this work is composed of 310 images, obtained from two CT scans of 31 individuals older than 20-years-old from the archives of the Hospital Estadual de Bauru, Bauru, São Paulo, Brazil. Each CT scan is composed by a sequence of five images. Fig. 14 shows three sequences (columns) of five images obtained from CT scans of three distinct individuals of our FSCT database.

\subsection{Frontal sinus segmentation}

As already mentioned, the performance of the automatic frontal sinus segmentation method proposed in this work was assessed by means of the Cohens Kappa coefficient computed between the automatic and the manual (ground truth) segmentation of all images of our FSCT database. Fig. 15 shows the coefficients obtained for all the images of our database. The average Kappa coefficient for all images was $\overline{x_{i}}=0.8852$, with a standard deviation $\sigma=0.05$. This good result shows the efficacy of the proposed automatic Frontal Sinus segmentation method.

\subsection{Frontal sinus recognition}

The assessment of the proposed frontal sinus recognition method based on CT images was carried out by analysing the EER, the ROC curve, and the CMC curve obtained in our experiments on the frontal sinus computed tomography (FSCT) database.

Fig. 16 shows the genuine and impostor score matching distributions obtained using the BAS shape descriptors and the protocol defined in Section 4.3.

Fig. 17 shows the ROC curve. The EER value was equal to $8.89 \%$, which can be considered a good result.

Fig. 18 shows the CMC curve. At rank 1, the identification accuracy was $77.25 \%$, at rank 5 , the identification accuracy was $86.91 \%$. The identification accuracy of $100 \%$ was reached at rank 12 . These results also can be considered good for this application.

\section{Conclusion}

The new method proposed in this work for automatic frontal sinus recognition based on computed tomography images obtained results that corroborate the results of other works in literature that affirm that frontal sinus can be successfully used for person identification.

Regarding the frontal sinus segmentation, the automatic segmentation proposed in our work is worth since the manual segmentation of the frontal sinus structure from radiographic images demands knowledge, hand ability, concentration, and time. The automated frontal sinus segmentation from CT images proposed in this work makes the process faster and more reliable.

The manual segmentation is a time consuming task. In our experiments, the average time needed to perform automatic segmentation of five images per CT scan was $30 \mathrm{~s}$, while to perform the manual segmentation, by a person that was previously trained for this task, it was necessary more than 15 min. Besides, during the frontal sinus manual segmentation that was performed under the supervision of an expert in Radiology, it was necessary to redo some segmentations. Therefore, the exhaustive, boring and unreliable task of manual segmentation can be replaced by the automatic one.

It is important to emphasize that the fully automatic segmentation in computed tomography images, despite the high contrast of such images, is a complex image processing problem because the lack of a standard structure of the lower region of the frontal sinuses. This characteristic makes the definition of the bottom frontal sinus limit a challenging problem. However, comparing with X-ray images, the high quality of the computed tomography images makes the frontal sinus segmentation easier and more robust. In addition, the sequence of $n$ images provided by computed tomography scans makes the matching more reliable, since in CT $n$ genuine comparisons are executed, per scan, differently from the X-ray scan, which relies on only one image.

Regarding the frontal sinus recognition, the identification rates obtained by our new method are very promising, since at rank 1 the identification accuracy was $77.25 \%$, and at rank 5 the accuracy was $86.91 \%$. These values show the efficacy of the BAS (Beam Angle Statistics) shape features as frontal sinus descriptors. In future work we intend to fuse such features with geometrical features, usually adopted by the methods proposed in literature. So, this good result can be even better.

Regarding the use of CT images, because of the reduction of CT technology and storage costs, CT images have been more and more used in clinical evaluations of nasal sinus disease and head and neck surgery, resulting in an indispensable tool in the diagnosis and treatment of paranasal sinuses diseases [22]. Then, CT images will be more and more available in future.

We can conclude, therefore, that the automatic frontal sinus recognition based on characteristics extracted from computed tomography images is viable and effective, becoming an important alternative in biometric person identification systems, in which other traditional identification methods cannot be applied.

\section{Acknowledgements}

The authors thank CAPES for the financial support.

\section{References}

[1] A.K. Jain, P. Flynn, A.A. Ross, Handbook of Biometrics, Springer-Verlag New York, Inc., Secaucus, NJ, USA, 2007 ISBN 038771040X.

[2] J.-P. Tang, D.-Y. Hu, F.-H. Jiang, X.-J. Yu, Assessing forensic applications of the frontal sinus in a Chinese Han population, Forensic Sci. Int.183 (13)(2009) 104.e1-104.e3, doi: http://dx.doi.org/10.1016/j.forsciint.2008.10.017 ISSN 0379-0738.

[3] E. Tatlisumak, G.Y. Ovali, A. Aslan, M. Asirdizer, Y. Zeyfeoglu, S. Tarhan, Identification of unknown bodies by using CT images of frontal sinus, Forensic Sci. Int. 26 (2007) 42-48

[4] W.B. Saunders, Dorland's Illustrated Medical Dictionary, 25th ed., (2011) Philadelphia.

[5] E. Zuckerkandl, Anatomie normale et pathologique des fosses nasales: et de leurs annexes pneumatiques, vol. 1, Masson, Paris, 1895.

[6] W.L. Culbert, F.M. Law, Identification by comparison of roentgenograms: of nasal accessory sinuses and mastoid processes, J. Am. Med. Assoc. 88 (21) (1927) 1634-1636.

[7] A. Schuller, A note on the identification of skulls by X-ray pictures of the frontal sinuses, Med. J. Aust. 1 (1943) 554-557.

[8] F.d.A.Q. Ribeiro, Standardized measurements of radiographic films of the frontal sinuses: an aid to identifying unknown persons, Ear Nose Throat J. 79 (1) $(2000) 26$

[9] N.J. Kirk, R.E. Wood, M. Goldstein, Skeletal identification using the frontal sinus region: a retrospective study of 39 cases, J. Forensic Sci. (2002).

[10] M.D.V.B.G. Brogdon, Forensic Radiology, CRC Press, 1998.

[11] C.B.R.B. Soares, M.S.C. Almeida, P. de Medeiros Loureiro Lopes, R.V. Belträo, A. dos Anjos Pontual, F.M. de Moraes Ramos-Perez, J.N. Figueroa, M.L. dos Anjos Pontual, Human identification study by means of frontal sinus imaginological aspects, Forensic Sci. Int. 262 (2016) 183-189, doi:http://dx.doi.org/10.1016/j. forsciint.2016.03.030 ISSN 0379-0738.

[12] N. Hashim, N. Hemalatha, K. Thangaraj, A. Kareem, A. Ahmed, N.F.N. Hassan, P. T.A. Jayaprakash, Practical relevance of prescribing superimposition for determining a frontal sinus pattern match, Forensic Sci. Int. 253 (2015) 137e1-137e7.

[13] K.A. Rabelo, M.L.A. Pontual, N.Q.J. ao, K.M. Paiva, F.M.M. Ramos-Perez, M.S. Santos, A.A. Pontual, Human identification by FSS system adapted to cephalometric radiographs, Forensic Sci. Int. 262 (2016) 232-277.

[14] C. Belaldavar, V.S. Kotrashetti, S.R. Hallikerimath, A.D. Kale, Assessment of frontal sinus dimensions to determine sexual dimorphism among Indian adults, J. Forensic Dent. Sci. 6 (1) (2014) 25-30.

[15] A.T. Uthman, N.H. Al-Hawi, A.S. Al-Naaimi, A.S. Tawfeeq, E.H. Suhail, Evaluation of frontal sinus and skull measurements using spiral CT scanning: an aid in unknown person identification, J. Forensic Sci. 56 (2) (2009) 403-408.

[16] S.P.M. Carvalho, R.H.A. Silva, C. Lopes-Júnior, A.S. Peres, A utilização de imagens na identificação humana em Odontologia Legal, Radiol. Bras. 42 (2) (2009) 125-130. 
[17] T.A. Xavier, A.S.S.D. Terada, R.H.A. Silva, Forensic application of the frontal and maxillary sinuses: a literature review, J. Forensic Radiol. Imaging 3 (2015) 105-110.

[18] N. Arica, F.T.Y. Vural, BAS: a perceptual shape descriptor based on the beam angle statistics, Pattern Recognit. Lett. 24 (2003) 1627-1639.

[19] G.B. Souza, A.N. Marana, Frontal Sinus Recognition: A Comparison Among Different Shape Descriptors, WVC - Workshop de Vis ao Computacional, (2013)
[20] G.B.D. Souza, A.N. Marana, HTS and HTSn: new shape descriptors based on Hough transform statistics, Comput. Vis. Image Underst. 127 (2014) 43-56, doi:http://dx.doi.org/10.1016/j.cviu.2014.06.010 ISSN 1077-3142.

[21] J. Cohen, A coefficient of agreement for nominal scales, Educ. Psychol. Meas. 20 (1960) 37-46.

[22] A.A. Taha, A. Hanbury, Metrics for evaluating 3D medical image segmentation: analysis, selection and tool, BMC Medical Imaging (2015) 15-29. 